QUARTERLY OF APPLIED MATHEMATICS

VOLUME LXV, NUMBER 1

MARCH 2007, PAGES 25-42

$\mathrm{S} 0033-569 \mathrm{X}(07) 01046-1$

Article electronically published on January 2, 2007

\title{
ON THE MIXED PROBLEM FOR HARMONIC FUNCTIONS IN A 2-D EXTERIOR CRACKED DOMAIN WITH NEUMANN CONDITION ON CRACKS
}

\author{
BY \\ P. A. KRUTITSKII \\ KIAM, Department 25, Miusskaya Sq. 4, Moscow 125047, Russia
}

\begin{abstract}
The mixed Dirichlet-Neumann problem for the Laplace equation in an unbounded plane domain with cuts (cracks) is studied. The Dirichlet condition is given on closed curves making up the boundary of the domain, while the Neumann condition is specified on the cuts. The existence of a classical solution is proved by potential theory and a boundary integral equation method. The integral representation for a solution is obtained in the form of potentials. The density of the potentials satisfies a uniquely solvable Fredholm integral equation of the second kind and index zero. Singularities of the gradient of the solution at the tips of the cuts are investigated.
\end{abstract}

1. Introduction. The boundary of a 2-D cracked domain consists of both closed curves and open arcs (cuts). Open arcs model cracks in solids and screens or wings in fluids. Different physical processes in cracked domains can be described by boundary value problems for the Laplace equation, for example, distribution of stationary heat and electric fields in cracked solids, electric flow in cracked semiconductors, flow of an ideal fluid over several obstacles and wings, etc. Appropriate boundary conditions must be specified on the total boundary, i.e., on both closed curves and open arcs (cracks). The Neumann boundary condition reflects the nonflow (of fluid, electric current, etc.) through the boundary. The Dirichlet boundary condition corresponds to the given temperature in heat theory, fluid pressure in hydrodynamics, electric potential in electrostatics, etc.

Boundary value problems with mixed boundary conditions were not treated in cracked domains by rigorous mathematical methods before. Even in the case of Laplace and Helmholtz equations the problems in domains bounded by closed curves [2], 13]-17] and problems in the exterior of cuts (cracks) [14, 16, [18,- 20] were treated separately, because different methods were used in their analysis. Previously the Neumann problem in the exterior of a cut was reduced to a hypersingular integral equation [14, 16, 18, 19] or

Received October 27, 2005.

2000 Mathematics Subject Classification. Primary 35J05, 35J25.

Key words and phrases. Laplace equation, Dirichlet-Neumann problem, boundary integral equation method.

(C)2007 Brown University 
to an infinite algebraic system of equations [20, while the Dirichlet problem in domains bounded by closed curves was reduced to the Fredholm equation of the second kind [13[17. The combination of these methods in the case of domains bounded by closed curves and cuts leads to an integral equation, which is algebraic or hypersingular on cuts, while it is an equation of the second kind with compact integral operators on the closed curves. The integral equation on the whole boundary is rather complicated to be effectively studied by standard methods. The approach suggested in the present paper enables us to reduce the mixed Dirichlet-Neumann problem in a cracked domain to the Fredholm integral equation of the second kind and index zero on the whole boundary with the help of a nonclassical angular potential. It is shown that the Fredholm integral equation is uniquely solvable; therefore the integral equation can be computed by a standard code by discretization and inversion of the matrix. So our approach is constructive, because it gives the way for finding the numerical solution for a mixed boundary value problem with complicated boundary in an exterior domain. Our approach is based on [5]-[6], where the problems in the exterior of cuts were reduced to the Fredholm integral equations using the angular potential. At first these problems were reduced to the Cauchy singular integral equation with additional conditions. Next, the singular integral equation was reduced to the Fredholm integral equation of the 2nd kind and index zero by regularization. In [7]-10] our approach has been applied to the Dirichlet and Neumann problems for the Laplace and Helmholtz equation in cracked domains. Some nonlinear problems of fluid dynamics were studied in 4. Using an integral representation for a solution of our problem in the form of potentials, obtained in the present paper, we derive explicit asymptotic formulas for singularities of the gradient of the solution at the tips of the cuts (cracks).

2. Formulation of the problem. By a simple open curve we mean a nonclosed smooth arc of finite length without self-intersections [16.

In the plane $x=\left(x_{1}, x_{2}\right) \in R^{2}$ we consider the exterior multiply connected domain bounded by simple open curves $\Gamma_{1}^{1}, \ldots, \Gamma_{N_{1}}^{1} \in C^{2, \lambda}$ and simple closed curves $\Gamma_{1}^{2}, \ldots, \Gamma_{N_{2}}^{2} \in$ $C^{1, \lambda}, \lambda \in(0,1]$, so that the curves have no points in common. We put

$$
\Gamma^{1}=\bigcup_{n=1}^{N_{1}} \Gamma_{n}^{1}, \quad \Gamma^{2}=\bigcup_{n=1}^{N_{2}} \Gamma_{n}^{2}, \quad \Gamma=\Gamma^{1} \cup \Gamma^{2} .
$$

The exterior connected domain bounded by $\Gamma^{2}$ will be called $\mathcal{D}$. We assume that each curve $\Gamma_{n}^{k}$ is parametrized by the arc length $s$ :

$$
\Gamma_{n}^{k}=\left\{x: x=x(s)=\left(x_{1}(s), x_{2}(s)\right), s \in\left[a_{n}^{k}, b_{n}^{k}\right]\right\}, n=1, \ldots, N_{k}, \quad k=1,2,
$$

so that $a_{1}^{1}<b_{1}^{1}<\ldots<a_{N_{1}}^{1}<b_{N_{1}}^{1}<a_{1}^{2}<b_{1}^{2}<\ldots<a_{N_{2}}^{2}<b_{N_{2}}^{2}$ and the domain $\mathcal{D}$ is on the right when the parameter $s$ increases on $\Gamma_{n}^{2}$. Therefore points $x \in \Gamma$ and values of the parameter $s$ are in one-to-one correspondence except $a_{n}^{2}, b_{n}^{2}$, which correspond to the same point $x$ for $n=1, \ldots, N_{2}$. The sets of intervals on the $O s$ axis

$$
\bigcup_{n=1}^{N_{1}}\left[a_{n}^{1}, b_{n}^{1}\right], \bigcup_{n=1}^{N_{2}}\left[a_{n}^{2}, b_{n}^{2}\right], \bigcup_{k=1}^{2} \bigcup_{n=1}^{N_{k}}\left[a_{n}^{k}, b_{n}^{k}\right]
$$


will be denoted by the same symbols as the corresponding sets of curves, that is, by $\Gamma^{1}$, $\Gamma^{2}$ and $\Gamma$ respectively.

We put $C^{0}\left(\Gamma_{n}^{2}\right)=\left\{\mathcal{F}(s): \mathcal{F}(s) \in C^{0}\left[a_{n}^{2}, b_{n}^{2}\right], \mathcal{F}\left(a_{n}^{2}\right)=\mathcal{F}\left(b_{n}^{2}\right)\right\}$, and

$$
C^{0}\left(\Gamma^{2}\right)=\bigcap_{n=1}^{N_{2}} C^{0}\left(\Gamma_{n}^{2}\right) .
$$

By $\mathcal{D}_{n}$ we denote the interior domain bounded by the curve $\Gamma_{n}^{2}$ for $n=1, \ldots, N_{2}$.

The tangent vector to $\Gamma$ at the point $x(s)$ we denote by $\tau_{x}=(\cos \alpha(s), \sin \alpha(s))$, where $\cos \alpha(s)=x_{1}^{\prime}(s), \sin \alpha(s)=x_{2}^{\prime}(s)$. Let $\mathbf{n}_{x}=(\sin \alpha(s),-\cos \alpha(s))$ be the normal vector to $\Gamma$ at $x(s)$. The direction of $\mathbf{n}_{x}$ is chosen such that it will coincide with the direction of $\tau_{x}$ if $\mathbf{n}_{x}$ is rotated counterclockwise through an angle of $\pi / 2$. Therefore $\mathbf{n}_{x}$ is the inward normal for $\mathcal{D}$ on $\Gamma^{2}$.

We consider the curves $\Gamma^{1}$ as a set of cuts. The side of $\Gamma^{1}$ which is on the left, when the parameter $s$ increases, will be denoted by $\left(\Gamma^{1}\right)^{+}$, and the opposite side will be denoted by $\left(\Gamma^{1}\right)^{-}$.

We say that the function $u(x)$ belongs to the smoothness class $\mathbf{K}$ if

1) $u \in C^{0}\left(\overline{\mathcal{D} \backslash \Gamma^{1}}\right) \cap C^{2}\left(\mathcal{D} \backslash \Gamma^{1}\right)$,

2) $\nabla u \in C^{0}\left(\overline{\mathcal{D} \backslash \Gamma^{1}} \backslash \Gamma^{2} \backslash X\right)$, where $X$ is a point-set, consisting of the end-points of $\Gamma^{1}$ :

$$
X=\bigcup_{n=1}^{N_{1}}\left(x\left(a_{n}^{1}\right) \cup x\left(b_{n}^{1}\right)\right),
$$

3 ) in the neighbourhood of any point $x(d) \in X$ for some constants $\mathcal{C}>0, \epsilon>-1$ the following inequality holds:

$$
|\nabla u| \leq \mathcal{C}|x-x(d)|^{\epsilon},
$$

where $x \rightarrow x(d)$ and $d=a_{n}^{1}$ or $d=b_{n}^{1}, \quad n=1, \ldots, N_{1}$.

REMARK. In the definition of the class $\mathbf{K}$ we consider $\Gamma^{1}$ as a set of cuts. In particular, by $C^{0}\left(\overline{\mathcal{D} \backslash \Gamma^{1}}\right)$ we denote a class of functions, which are continuously extended on the cuts $\Gamma^{1}$ from the left and right and are continuous at the tips of the cuts $\Gamma^{1}$. However, values of these functions on $\Gamma^{1}$ from the left and right can be different everywhere except at the tips, so that the functions may have a jump on $\Gamma^{1}$.

Let us formulate the mixed Dirichlet-Neumann problem for the Laplace equation in the domain $\mathcal{D} \backslash \Gamma^{1}$.

Problem $\mathbf{U}$. Find a function $u(x)$ of class $\mathbf{K}$ so that $u(x)$ satisfies the Laplace equation

$$
u_{x_{1} x_{1}}(x)+u_{x_{2} x_{2}}(x)=0, \quad x \in \mathcal{D} \backslash \Gamma^{1},
$$

the boundary conditions

$$
\begin{aligned}
\left.\frac{\partial u(x)}{\partial \mathbf{n}_{x}}\right|_{x(s) \in\left(\Gamma^{1}\right)^{+}}= & F^{+}(s),\left.\quad \frac{\partial u(x)}{\partial \mathbf{n}_{x}}\right|_{x(s) \in\left(\Gamma^{1}\right)^{-}}=F^{-}(s), \\
& \left.u(x(s))\right|_{\Gamma^{2}}=F(s),
\end{aligned}
$$

and the following conditions as $|x|=\sqrt{x_{1}^{2}+x_{2}^{2}} \rightarrow \infty$ :

$$
|u(x)| \leq \text { const }, \quad|\nabla u|=o\left(|x|^{-1}\right) .
$$

All conditions of the problem $\mathbf{U}$ must be satisfied in the classical sense. 
The edge condition (1) ensures the absence of point sources at the ends of $\Gamma^{1}$. It is assumed that $N_{2} \geq 1$. If $N_{1}=0$ and the cuts $\Gamma^{1}$ are absent, then the problem $\mathbf{U}$ transforms to the classical Dirichlet problem in an exterior domain $\mathcal{D}$ without cuts.

Using the energy equalities we can prove the following assertion.

TheOrem 1. The problem $\mathbf{U}$ has at most one solution.

By $\int_{\Gamma^{k}} \ldots d \sigma$ we mean

$$
\sum_{n=1}^{N_{k}} \int_{a_{n}^{k}}^{b_{n}^{k}} \ldots d \sigma
$$

Proof. Consider the homogeneous problem $\mathbf{U}$ and assume that $u_{0}(x)$ is a solution of the homogeneous problem (with $F^{ \pm}(s) \equiv 0, F(s) \equiv 0$ ). Our aim is to show that $u_{0}(x) \equiv 0$. Since $\Gamma^{2} \in C^{1, \lambda}$ and $u_{0}(x) \in \mathbf{K}$, from [3, Remark to Lemma 6.18] we have $u_{0}(x) \in C^{1}\left(\overline{\mathcal{D}} \backslash \Gamma^{1}\right)$ (see also [16, Remark to Section 65.4]). Combining this result with the smoothness ensured for $u_{0}(x)$ by the class $\mathbf{K}$, we have $\nabla u_{0}(x) \in C^{0}\left(\overline{\mathcal{D} \backslash \Gamma^{1}} \backslash X\right)$, and inequality (1) holds at the tips of $\Gamma^{1}$. We envelop each cut $\Gamma_{n}^{1}\left(n=1, \ldots, N_{1}\right)$ by a closed contour so that all contours lie in $\mathcal{D} \backslash \Gamma^{1}$. Next we write the energy equalities for a domain, bounded by our auxiliary contours, $\Gamma^{2}$ and the circle of a large enough radius $r$. We allow the auxiliary contours to shrink to $\Gamma^{1}$ and let $r$ tend to infinity. Using the conditions at infinity $(2 \mathrm{~b})$ and the smoothness of $u_{0}(x)$ established above, we obtain

$$
\left\|\nabla u_{0}\right\|_{L_{2}\left(\mathcal{D} \backslash \Gamma^{1}\right)}^{2}=\int_{\Gamma^{1}}\left[u_{0}^{+}\left(\frac{\partial u_{0}}{\partial \mathbf{n}_{x}}\right)^{+}-u_{0}^{-}\left(\frac{\partial u_{0}}{\partial \mathbf{n}_{x}}\right)^{-}\right] d s-\int_{\Gamma^{2}} u_{0} \frac{\partial u_{0}}{\partial \mathbf{n}_{x}} d s .
$$

Taking into account the homogeneous boundary conditions (2a), we have

$$
\left\|\nabla u_{0}\right\|_{L_{2}\left(\mathcal{D} \backslash \Gamma^{1}\right)}^{2}=0 .
$$

Hence $u_{0}(x) \equiv$ const and const $=0$ due to the homogeneous Dirichlet boundary condition on $\Gamma^{2}$. Therefore $u_{0}(x) \equiv 0$, and the theorem is proved thanks to the linearity of the problem $\mathbf{U}$.

3. Integral equations at the boundary. Below we assume that

$$
F^{+}(s), F^{-}(s) \in C^{0, \lambda}\left(\Gamma^{1}\right), \quad F(s) \in C^{0}\left(\Gamma^{2}\right), \quad \lambda \in(0,1] .
$$

Note that the Hölder exponent $\lambda$ in the description of smoothness of these functions and in the description of smoothness of the boundary $\Gamma$ is the same. If the exponents are different in practice, then by $\lambda$ we denote the least.

If $\mathcal{B}_{1}\left(\Gamma^{1}\right), \mathcal{B}_{2}\left(\Gamma^{2}\right)$ are Banach spaces of functions given on $\Gamma^{1}$ and $\Gamma^{2}$, then for functions given on $\Gamma$ we introduce the Banach space $\mathcal{B}_{1}\left(\Gamma^{1}\right) \cap \mathcal{B}_{2}\left(\Gamma^{2}\right)$ with the norm $\|\cdot\|_{\mathcal{B}_{1}\left(\Gamma^{1}\right) \cap \mathcal{B}_{2}\left(\Gamma^{2}\right)}=\|\cdot\|_{\mathcal{B}_{1}\left(\Gamma^{1}\right)}+\|\cdot\|_{\mathcal{B}_{2}\left(\Gamma^{2}\right)}$. An example of such a Banach space is $C^{0}(\Gamma)=$ $C^{0}\left(\Gamma^{1}\right) \cap C^{0}\left(\Gamma^{2}\right)$.

We shall construct the solution of the problem $\mathbf{U}$ from the smoothness class $\mathbf{K}$ with the help of potential theory for harmonic functions. 
We consider an angular potential [1, 5, 12, Appendix] for the Laplace equation:

$$
w_{1}[\mu](x)=-\frac{1}{2 \pi} \int_{\Gamma^{1}} \mu(\sigma) V(x, y(\sigma)) d \sigma .
$$

The kernel $V(x, y(\sigma)$ ) is defined (up to indeterminacy $2 \pi m, m= \pm 1, \pm 2, \ldots$ ) by the formulae

where

$$
\cos V(x, y(\sigma))=\frac{x_{1}-y_{1}(\sigma)}{|x-y(\sigma)|}, \quad \sin V(x, y(\sigma))=\frac{x_{2}-y_{2}(\sigma)}{|x-y(\sigma)|},
$$

$$
y=y(\sigma)=\left(y_{1}(\sigma), y_{2}(\sigma)\right) \in \Gamma^{1},|x-y(\sigma)|=\sqrt{\left(x_{1}-y_{1}(\sigma)\right)^{2}+\left(x_{2}-y_{2}(\sigma)\right)^{2}} .
$$

One can see that $V(x, \sigma)$ is the angle between the vector $\overrightarrow{y(\sigma) x}$ and the direction of the $O x_{1}$ axis. More precisely, $V(x, y(\sigma))$ is a many-valued harmonic function conjugate to $\ln |x-y(\sigma)|$.

Below by $V(x, y(\sigma))$ we denote an arbitrary fixed branch of this function, which varies continuously with $\sigma$ along each curve $\Gamma_{n}^{1}\left(n=1, \ldots, N_{1}\right)$ for given fixed $x \notin \Gamma^{1}$.

Under this definition of $V(x, y(\sigma))$, the potential $w_{1}[\mu](x)$ is a many-valued function. In order that the potential $w_{1}[\mu](x)$ be single-valued, it is necessary to impose the following additional conditions:

$$
\int_{a_{n}^{1}}^{b_{n}^{1}} \mu(\sigma) d \sigma=0, \quad n=1, \ldots, N_{1} .
$$

Below we suppose that the density $\mu(\sigma)$ belongs to the Banach space $C_{q}^{\omega}\left(\Gamma^{1}\right), \omega \in$ $(0,1], q \in[0,1)$ and satisfies conditions (5).

We say that $\mu(s) \in C_{q}^{\omega}\left(\Gamma^{1}\right)$ if

$$
\mu(s) \prod_{n=1}^{N_{1}}\left|s-a_{n}^{1}\right|^{q}\left|s-b_{n}^{1}\right|^{q} \in C^{0, \omega}\left(\Gamma^{1}\right),
$$

where $C^{0, \omega}\left(\Gamma^{1}\right)$ is a Hölder space with exponent $\omega$ and

$$
\|\mu(s)\|_{C_{q}^{\omega}\left(\Gamma^{1}\right)}=\left\|\mu(s) \prod_{n=1}^{N_{1}}\left|s-a_{n}^{1}\right|^{q}\left|s-b_{n}^{1}\right|^{q}\right\|_{C^{0, \omega}\left(\Gamma^{1}\right)} .
$$

As shown in [1], [5, [12, Appendix], for such $\mu(\sigma)$ the angular potential $w_{1}[\mu](x)$ belongs to the class $\mathbf{K}$. In particular, the inequality (1) holds with $\epsilon=-q$ if $q \in(0,1)$. Moreover, integrating $w_{1}[\mu](x)$ by parts and using (5), we express the angular potential in terms of a double layer potential

$$
w_{1}[\mu](x)=\frac{1}{2 \pi} \int_{\Gamma^{1}} \rho(\sigma) \frac{\partial}{\partial \mathbf{n}_{y}} \ln |x-y(\sigma)| d \sigma,
$$

with the density

$$
\rho(\sigma)=\int_{a_{n}^{1}}^{\sigma} \mu(\xi) d \xi, \quad \sigma \in\left[a_{n}^{1}, b_{n}^{1}\right], \quad n=1, \ldots, N_{1} .
$$


Consequently, $w_{1}[\mu](x)$ satisfies the Laplace equation outside $\Gamma^{1}$ and the conditions at infinity $(2 b)$.

Let us construct a solution of the problem $\mathbf{U}$. We seek a solution of the problem in the following form:

$$
u[\nu, \mu](x)=v_{1}[\nu](x)+w[\mu](x)+h[\nu, \mu](x),
$$

where

$$
\begin{gathered}
w[\mu](x)=w_{1}[\mu](x)+w_{2}[\mu](x), \\
v_{1}[\nu](x)=-\frac{1}{2 \pi} \int_{\Gamma^{1}} \nu(\sigma) \ln |x-y(\sigma)| d \sigma, \\
w_{2}[\mu](x)=-\frac{1}{2 \pi} \int_{\Gamma^{2}} \mu(\sigma) \frac{\partial}{\partial \mathbf{n}_{y}} \ln |x-y(\sigma)| d \sigma,
\end{gathered}
$$

and $w_{1}[\mu](x)$ is given by (4). By $h[\nu, \mu](x)$ we denote the sum of point sources placed at the fixed points $Y_{k}$ lying inside $\Gamma_{k}^{2}\left(k=1, \ldots, N_{2}\right)$ and a constant:

$$
\begin{aligned}
h[\nu, \mu](x)= & -\frac{1}{2 \pi} \sum_{k=2}^{N_{2}} \int_{\Gamma_{k}^{2}} \mu(\sigma) d \sigma \ln \left|x-Y_{k}\right| \\
& +\frac{1}{2 \pi}\left[\int_{\Gamma^{2}} \mu(\sigma) d \sigma+\int_{\Gamma^{1}} \nu(\sigma) d \sigma-\int_{\Gamma_{1}^{2}} \mu(\sigma) d \sigma\right] \ln \left|x-Y_{1}\right|+\int_{\Gamma^{2}} \mu(\sigma) d \sigma \\
= & \frac{1}{2 \pi} \int_{\Gamma^{1}} \nu(\sigma) d \sigma \ln \left|x-Y_{1}\right|+h_{2}[\mu](x) .
\end{aligned}
$$

Here

$$
\begin{gathered}
h_{2}[\mu](x)=-\frac{1}{2 \pi} \sum_{k=2}^{N_{2}} \int_{\Gamma_{k}^{2}} \mu(\sigma) d \sigma \ln \left|x-Y_{k}\right| \\
+\frac{1}{2 \pi}\left[\int_{\Gamma^{2}} \mu(\sigma) d \sigma-\int_{\Gamma_{1}^{2}} \mu(\sigma) d \sigma\right] \ln \left|x-Y_{1}\right|+\int_{\Gamma^{2}} \mu(\sigma) d \sigma ; \quad Y_{k} \in \mathcal{D}_{k}, \quad k=1, \ldots, N_{2} .
\end{gathered}
$$

Clearly, $h[\nu, \mu](x)$ obeys the Laplace equation in $R^{2} \backslash \bigcup_{k=1}^{N_{2}} Y_{k}$ and belongs to

$$
C^{\infty}\left(R^{2} \backslash \bigcup_{k=1}^{N_{2}} Y_{k}\right)
$$

Furthermore, if $x(s) \in \Gamma$, then $h[\nu, \mu](x(s)) \in C^{1, \lambda}(\Gamma)$ in $s$. We need the function $h[\nu, \mu](x)$ to construct a uniquely solvable integral equation. Moreover, $h[\nu, \mu](x)$ is taken in such a way that $u[\nu, \mu](x)$ in (6) satisfies conditions (2b) at infinity.

We will look for the density $\nu(\sigma)$ in the space $C^{0, \lambda}\left(\Gamma^{1}\right)$. 
We will seek $\mu(s)$ in the Banach space $C_{q}^{\omega}\left(\Gamma^{1}\right) \cap C^{0}\left(\Gamma^{2}\right), \quad \omega \in(0,1], \quad q \in[0,1)$ with the norm $\|\cdot\|_{C_{q}^{\omega}\left(\Gamma^{1}\right) \cap C^{0}\left(\Gamma^{2}\right)}=\|\cdot\|_{C_{q}^{\omega}\left(\Gamma^{1}\right)}+\|\cdot\|_{C^{0}\left(\Gamma^{2}\right)}$. Furthermore, $\mu(s)$ must satisfy conditions (5).

It follows from [1, 5, 17, 12, Appendix] that for such $\mu(s), \nu(s)$ the function (6) belongs to the class $\mathbf{K}$ and satisfies all the conditions of the problem $\mathbf{U}$ except the boundary conditions (2a).

To satisfy the boundary conditions, we insert (6) in (2a), use limit formulas for the normal derivative of the angular potential [1, 5, [12, Appendix] and arrive at the system of integral equations for the densities $\mu(s), \nu(s)$ :

$$
\begin{gathered}
\pm \frac{1}{2} \nu(s)+\frac{1}{2 \pi} \int_{\Gamma^{1}} \nu(\sigma) \frac{\cos \varphi_{0}(x(s), y(\sigma))}{|x(s)-y(\sigma)|} d \sigma \\
-\frac{1}{2 \pi} \int_{\Gamma^{1}} \mu(\sigma) \frac{\sin \varphi_{0}(x(s), y(\sigma))}{|x(s)-y(\sigma)|} d \sigma \\
-\frac{1}{2 \pi} \int_{\Gamma^{2}} \mu(\sigma) \frac{\partial}{\partial \mathbf{n}_{x}} \frac{\partial}{\partial \mathbf{n}_{y}} \ln |x(s)-y(\sigma)| d \sigma+\frac{\partial}{\partial \mathbf{n}_{x}} h[\nu, \mu](x(s))=F^{ \pm}(s), \quad s \in \Gamma^{1}, \\
-\frac{1}{2 \pi} \int_{\Gamma^{1}} \mu(\sigma) V(x(s), y(\sigma)) d \sigma-\frac{1}{2 \pi} \int_{\Gamma^{1}} \nu(\sigma) \ln |x(s)-y(\sigma)| d \sigma \\
+\frac{1}{2} \mu(s)-\frac{1}{2 \pi} \int_{\Gamma^{2}} \mu(\sigma) \frac{\partial}{\partial \mathbf{n}_{y}} \ln |x(s)-y(\sigma)| d \sigma+h[\nu, \mu](x(s))=F(s), \quad s \in \Gamma^{2} .
\end{gathered}
$$

By $\varphi_{0}(x, y)$ we denote the angle between the vector $\overrightarrow{x y}$ and the direction of the normal $\mathbf{n}_{x}$. The angle $\varphi_{0}(x, y)$ is taken to be positive if it is measured counterclockwise from $\mathbf{n}_{x}$ and negative if it is measured clockwise from $\mathbf{n}_{x}$. Also, $\varphi_{0}(x, y)$ is continuous in $x, y \in \Gamma$ if $x \neq y$. Note that for $x(s), y(\sigma) \in \Gamma$ and $x \neq y$ we have the relationships

$$
\begin{gathered}
\frac{\partial}{\partial \mathbf{n}_{x}} \ln |x(s)-y(\sigma)|=\frac{\partial}{\partial \tau_{x}} V(x(s), y(\sigma))=\frac{\partial}{\partial s} V(x(s), y(\sigma)) \\
=-\frac{\cos \varphi_{0}(x(s), y(\sigma))}{|x(s)-y(\sigma)|}=-\frac{\sin (V(x(s), y(\sigma))-\alpha(s))}{|x(s)-y(\sigma)|}, \\
\frac{\partial}{\partial \mathbf{n}_{x}} V(x(s), y(\sigma))=-\frac{\partial}{\partial \tau_{x}} \ln |x(s)-y(\sigma)|=-\frac{\partial}{\partial s} \ln |x(s)-y(\sigma)| \\
=\frac{\sin \varphi_{0}(x(s), y(\sigma))}{|x(s)-y(\sigma)|}=-\frac{\cos (V(x(s), y(\sigma))-\alpha(s))}{|x(s)-y(\sigma)|}
\end{gathered}
$$

where $\alpha(s)$ is the inclination of the tangent $\tau_{x}$ to the $O x_{1}$ axis, and $V(x, y(\sigma))$ is the kernel of the angular potential (4).

The second integral term in (8a) is a Cauchy singular integral. The kernel of the third integral term in (8b) has a weak singularity as $s=\sigma$.

Equation (8a) is obtained as $x \rightarrow x(s) \in\left(\Gamma^{1}\right)^{ \pm}$and comprises two integral equations. The upper sign denotes the integral equation on $\left(\Gamma^{1}\right)^{+}$, and the lower sign denotes the integral equation on $\left(\Gamma^{1}\right)^{-}$.

In addition to the integral equations written above we have conditions (5). 
Subtracting the integral equations (8a), we find

$$
\nu(s)=\left(F^{+}(s)-F^{-}(s)\right) \in C^{0, \lambda}\left(\Gamma^{1}\right) .
$$

We note that $\nu(s)$ is found completely and satisfies all required conditions. Hence, the potential $v_{1}[\nu](x)$ is found completely as well. Additionally,

$$
h[\nu, \mu](x)=\frac{1}{2 \pi} \int_{\Gamma^{1}}\left(F^{+}(\sigma)-F^{-}(\sigma)\right) d \sigma \ln \left|x-Y_{1}\right|+h_{2}[\mu](x),
$$

where $h_{2}[\mu](x)$ is given by $(7 \mathrm{~b})$.

We introduce the function $f(s)$ on $\Gamma$ by the formulas

$$
\begin{gathered}
f(s)=\frac{1}{2}\left(F^{+}(s)+F^{-}(s)\right) \\
-\frac{1}{2 \pi} \int_{\Gamma^{1}}\left(F^{+}(\sigma)-F^{-}(\sigma)\right) \frac{\cos \varphi_{0}(x(s), y(\sigma))}{|x(s)-y(\sigma)|} d \sigma \\
-\frac{1}{2 \pi} \int_{\Gamma^{1}}\left(F^{+}(\sigma)-F^{-}(\sigma)\right) d \sigma \frac{\partial}{\partial \mathbf{n}_{x}} \ln \left|x(s)-Y_{1}\right|, \quad s \in \Gamma^{1},
\end{gathered}
$$

and

$$
\begin{gathered}
f(s)=F(s)+\frac{1}{2 \pi} \int_{\Gamma^{1}}\left(F^{+}(\sigma)-F^{-}(\sigma)\right) \ln |x(s)-y(\sigma)| d \sigma \\
-\frac{1}{2 \pi} \int_{\Gamma^{1}}\left(F^{+}(\sigma)-F^{-}(\sigma)\right) d \sigma \ln \left|x(s)-Y_{1}\right|, \quad s \in \Gamma^{2},
\end{gathered}
$$

where $F^{ \pm}(s)$ and $F(s)$ are specified in (2a) and satisfy conditions (3). As is shown in [6], if $s \in \Gamma^{1}$, then $f(s) \in C^{0, \lambda}\left(\Gamma^{1}\right)$. Consequently,

$$
f(s) \in C^{0, \lambda}\left(\Gamma^{1}\right) \cap C^{0}\left(\Gamma^{2}\right) .
$$

Adding the integral equations (8a) we obtain the integral equation for $\mu(s)$ on $\Gamma^{1}$ :

$$
\begin{gathered}
-\frac{1}{2 \pi} \int_{\Gamma^{1}} \mu(\sigma) \frac{\sin \varphi_{0}(x(s), y(\sigma))}{|x(s)-y(\sigma)|} d \sigma \\
-\frac{1}{2 \pi} \int_{\Gamma^{2}} \mu(\sigma) \frac{\partial}{\partial \mathbf{n}_{x}} \frac{\partial}{\partial \mathbf{n}_{y}} \ln |x-y(\sigma)| d \sigma+\frac{\partial}{\partial \mathbf{n}_{x}} h_{2}[\mu](x(s))=f(s), \quad s \in \Gamma^{1},
\end{gathered}
$$

where $f(s)$ is given by (10a).

Equation (8b) on $\Gamma^{2}$ takes the form

$$
\begin{gathered}
-\frac{1}{2 \pi} \int_{\Gamma^{1}} \mu(\sigma) V(x(s), y(\sigma)) d \sigma \\
+\frac{1}{2} \mu(s)-\frac{1}{2 \pi} \int_{\Gamma^{2}} \mu(\sigma) \frac{\partial}{\partial \mathbf{n}_{y}} \ln |x(s)-y(\sigma)| d \sigma+h_{2}[\mu](x(s))=f(s), \quad s \in \Gamma^{2},
\end{gathered}
$$

where $f(s)$ is given in (10b). 
Thus, if $\mu(s)$ is a solution of equations (11), (5) from the space $C_{q}^{\omega}\left(\Gamma^{1}\right) \cap C^{0}\left(\Gamma^{2}\right)$ with $\omega \in(0,1], q \in[0,1)$, then the potential (6) with $\nu(s)$ from (9) satisfies all conditions of the problem $\mathbf{U}$ and belongs to the class $\mathbf{K}$.

The following theorem holds.

Theorem 2. Let $\Gamma^{1} \in C^{2, \lambda}, \Gamma^{2} \in C^{1, \lambda}$ and conditions (3) hold. If equations (11), (5) have a solution $\mu(s)$ from the Banach space $C_{q}^{\omega}\left(\Gamma^{1}\right) \cap C^{0}\left(\Gamma^{2}\right)$ for some $\omega \in(0,1]$ and $q \in[0,1)$, then the solution of the problem $\mathbf{U}$ exists, belongs to the class $\mathbf{K}$ and is given by $(6)$, where $\nu(s)$ is defined in (9).

If $s \in \Gamma^{2}$, then (11b) is an equation of the second kind with a weak singularity in the kernel. If $s \in \Gamma^{1}$, then (11a) is a Cauchy singular integral equation of the first kind [16].

Our further treatment will be aimed at the proof of the solvability of the system (11), (5) in the Banach space $C_{q}^{\omega}\left(\Gamma^{1}\right) \cap C^{0}\left(\Gamma^{2}\right)$. Moreover, we reduce the system (11), (5) to a Fredholm equation of the second kind and index zero, which can be easily computed by classical methods.

Equation (11b) on $\Gamma^{2}$ can be rewritten in the form

$$
\mu(s)+\int_{\Gamma} \mu(\sigma) A_{2}(s, \sigma) d \sigma=2 f(s), \quad s \in \Gamma^{2},
$$

where

$$
\begin{gathered}
A_{2}(s, \sigma)=\left\{-\frac{1}{\pi}\left(1-\delta\left(\Gamma^{2}, \sigma\right)\right) V(x(s), y(\sigma))\right. \\
-\frac{1}{\pi} \delta\left(\Gamma^{2}, \sigma\right) \frac{\partial}{\partial \mathbf{n}_{y}} \ln |x(s)-y(\sigma)|-\frac{1}{\pi} \sum_{k=2}^{N_{2}} \delta\left(\Gamma_{k}^{2}, \sigma\right) \ln \left|x(s)-Y_{k}\right| \\
\left.+\frac{1}{\pi}\left(\delta\left(\Gamma^{2}, \sigma\right)-\delta\left(\Gamma_{1}^{2}, \sigma\right)\right) \ln \left|x(s)-Y_{1}\right|+2 \delta\left(\Gamma^{2}, \sigma\right)\right\} .
\end{gathered}
$$

By $\delta(\gamma, \sigma)$ we denote the characteristic function of the set $\gamma$ :

$$
\delta(\gamma, \sigma)= \begin{cases}0, & \text { if } \sigma \notin \gamma \\ 1, & \text { if } \sigma \in \gamma .\end{cases}
$$

The kernel $A_{2}(s, \sigma)$ has a weak singularity if $s=\sigma \in \Gamma^{2}$, and $A_{2}(s, \sigma)$ is continuous if $s \neq \sigma \quad\left(s \in \Gamma^{2}, \sigma \in \Gamma\right)$.

Remark. Evidently, $f\left(a_{n}^{2}\right)=f\left(b_{n}^{2}\right)$ and $A_{2}\left(a_{n}^{2}, \sigma\right)=A_{2}\left(b_{n}^{2}, \sigma\right)$ for $\sigma \in \Gamma, \sigma \neq a_{n}^{2}, b_{n}^{2}$ $\left(n=1, \ldots, N_{2}\right)$. Hence, if $\mu(s)$ is a solution of equation (12) from $C^{0}\left(\bigcup_{n=1}^{N_{2}}\left[a_{n}^{2}, b_{n}^{2}\right]\right)$, then, according to the equality (12), $\mu(s)$ automatically satisfies the matching conditions $\mu\left(a_{n}^{2}\right)=\mu\left(b_{n}^{2}\right)$ for $n=1, \ldots, N_{2}$ and, therefore, belongs to $C^{0}\left(\Gamma^{2}\right)$. This observation can be helpful in finding numerical solutions, since we may discard the matching conditions $\mu\left(a_{n}^{2}\right)=\mu\left(b_{n}^{2}\right) \quad\left(n=1, \ldots, N_{2}\right)$, which are automatically fulfilled.

It can be easily proved that

$$
-\frac{\partial}{\partial s} \ln \frac{|x(s)-y(\sigma)|}{|s-\sigma|}=\frac{\sin \varphi_{0}(x(s), y(\sigma))}{|x(s)-y(\sigma)|}-\frac{1}{\sigma-s} \in C^{0, \lambda}\left(\Gamma^{1} \times \Gamma^{1}\right)
$$


(see [5, 6] for details). Therefore we can rewrite (11a) in the form

$$
\frac{1}{\pi} \int_{\Gamma^{1}} \mu(\sigma) \frac{d \sigma}{\sigma-s}+\int_{\Gamma} \mu(\sigma) M(s, \sigma) d \sigma=-2 f(s), \quad s \in \Gamma^{1},
$$

where

$$
\begin{gathered}
M(s, \sigma)=\frac{1}{\pi}\left\{\left(1-\delta\left(\Gamma^{2}, \sigma\right)\right)\left(\frac{\sin \varphi_{0}(x(s), y(\sigma))}{|x(s)-y(\sigma)|}-\frac{1}{\sigma-s}\right)\right. \\
+\delta\left(\Gamma^{2}, \sigma\right) \frac{\partial}{\partial \mathbf{n}_{x}}\left[\frac{\partial}{\partial \mathbf{n}_{y}} \ln |x(s)-y(\sigma)|+\sum_{k=2}^{N_{2}} \delta\left(\Gamma_{k}^{2}, \sigma\right) \ln \left|x(s)-Y_{k}\right|\right. \\
\left.\left.-\left(1-\delta\left(\Gamma_{1}^{2}, \sigma\right)\right) \ln \left|x(s)-Y_{1}\right|\right]\right\}
\end{gathered}
$$

and $M(s, \sigma) \in C^{0, \lambda}\left(\Gamma^{1} \times \Gamma\right)$.

4. The Fredholm integral equation and the solution of the problem. Inverting the singular integral operator in (13), we arrive at the following integral equation of the second kind [5, 6]:

$$
\mu(s)+\frac{1}{Q_{1}(s)} \int_{\Gamma} \mu(\sigma) A_{0}(s, \sigma) d \sigma+\frac{1}{Q_{1}(s)} \sum_{n=0}^{N_{1}-1} G_{n} s^{n}=\frac{1}{Q_{1}(s)} \Phi_{0}(s), \quad s \in \Gamma^{1},
$$

where

$$
\begin{gathered}
A_{0}(s, \sigma)=-\frac{1}{\pi} \int_{\Gamma^{1}} \frac{M(\xi, \sigma)}{\xi-s} Q_{1}(\xi) d \xi \\
Q_{1}(s)=\prod_{n=1}^{N_{1}}\left|\sqrt{s-a_{n}^{1}} \sqrt{b_{n}^{1}-s}\right| \operatorname{sign}\left(s-a_{n}^{1}\right), \\
\Phi_{0}(s)=\frac{1}{\pi} \int_{\Gamma^{1}} \frac{2 Q_{1}(\sigma) f(\sigma)}{\sigma-s} d \sigma,
\end{gathered}
$$

and $G_{0}, \ldots, G_{N_{1}-1}$ are arbitrary constants.

To derive equations for $G_{0}, \ldots, G_{N_{1}-1}$, we substitute $\mu(s)$ from (14) in the conditions (5). Then we obtain

$$
\int_{\Gamma} \mu(\sigma) l_{n}(\sigma) d \sigma+\sum_{m=0}^{N_{1}-1} B_{n m} G_{m}=H_{n}, \quad n=1, \ldots, N_{1}
$$


where

$$
\begin{gathered}
l_{n}(\sigma)=-\int_{\Gamma_{n}^{1}} Q_{1}^{-1}(s) A_{0}(s, \sigma) d s, \\
B_{n m}=-\int_{\Gamma_{n}^{1}} Q_{1}^{-1}(s) s^{m} d s, \\
H_{n}=-\int_{\Gamma_{n}^{1}} Q_{1}^{-1}(s) \Phi_{0}(s) d s .
\end{gathered}
$$

By $B$ we denote the $N_{1} \times N_{1}$ matrix with the elements $B_{n m}$ from (16). As shown in [6. Lemma 7], [1] the matrix $B$ is invertible. The elements of the inverse matrix will be called $\left(B^{-1}\right)_{n m}$. Inverting the matrix $B$ in (15), we express the constants $G_{0}, \ldots, G_{N_{1}-1}$ in terms of $\mu(s)$ as

$$
G_{n}=\sum_{m=1}^{N_{1}}\left(B^{-1}\right)_{n m}\left[H_{m}-\int_{\Gamma} \mu(\sigma) l_{m}(\sigma) d \sigma\right] .
$$

We substitute $G_{n}$ in (14) and obtain the following integral equation for $\mu(s)$ on $\Gamma^{1}$ :

$$
\mu(s)+\frac{1}{Q_{1}(s)} \int_{\Gamma} \mu(\sigma) A_{1}(s, \sigma) d \sigma=\frac{1}{Q_{1}(s)} \Phi_{1}(s), \quad s \in \Gamma^{1},
$$

where

$$
\begin{gathered}
A_{1}(s, \sigma)=A_{0}(s, \sigma)-\sum_{n=0}^{N_{1}-1} s^{n} \sum_{m=1}^{N_{1}}\left(B^{-1}\right)_{n m} l_{m}(\sigma), \\
\Phi_{1}(s)=\Phi_{0}(s)-\sum_{n=0}^{N_{1}-1} s^{n} \sum_{m=1}^{N_{1}}\left(B^{-1}\right)_{n m} H_{m} .
\end{gathered}
$$

It can be verified directly that any solution of (17) in the required space satisfies conditions (5) automatically. It can be shown using the properties of singular integrals [2, [16] that $\Phi_{0}(s), A_{0}(s, \sigma)$ are Hölder continuous functions if $s \in \Gamma^{1}, \sigma \in \Gamma$. Therefore, $\Phi_{1}(s), \quad A_{1}(s, \sigma)$ are also Hölder continuous functions if $s \in \Gamma^{1}, \sigma \in \Gamma$. Consequently, any solution of (17) belongs to $C_{1 / 2}^{\omega}\left(\Gamma^{1}\right)$, and below we look for $\mu(s)$ on $\Gamma^{1}$ in this space.

We put

$$
Q(s)=\left(1-\delta\left(\Gamma^{2}, s\right)\right) Q_{1}(s)+\delta\left(\Gamma^{2}, s\right), \quad s \in \Gamma .
$$

Instead of $\mu(s) \in C_{1 / 2}^{\omega}\left(\Gamma^{1}\right) \cap C^{0}\left(\Gamma^{2}\right)$ we introduce the new unknown function $\mu_{*}(s)=$ $\mu(s) Q(s) \in C^{0, \omega}\left(\Gamma^{1}\right) \cap C^{0}\left(\Gamma^{2}\right)$ and rewrite (12), (17) in the form of one equation:

$$
\mu_{*}(s)+\int_{\Gamma} \mu_{*}(\sigma) Q^{-1}(\sigma) A(s, \sigma) d \sigma=\Phi(s), \quad s \in \Gamma,
$$

where

$$
\begin{gathered}
A(s, \sigma)=\left(1-\delta\left(\Gamma^{2}, s\right)\right) A_{1}(s, \sigma)+\delta\left(\Gamma^{2}, s\right) A_{2}(s, \sigma), \\
\Phi(s)=\left(1-\delta\left(\Gamma^{2}, s\right)\right) \Phi_{1}(s)+2 \delta\left(\Gamma^{2}, s\right) f(s) .
\end{gathered}
$$


Thus, the system of equations (5), (11) for $\mu(s)$ has been reduced to the equation (18) for the function $\mu_{*}(s)$. It is clear from our consideration that any solution of (18) gives a solution of system (5), (11) and conversely.

As noted above, $\Phi_{1}(s)$ and $A_{1}(s, \sigma)$ are Hölder continuous functions if $s \in \Gamma^{1}, \sigma \in \Gamma$. More precisely (see [6]), $A_{1}(s, \sigma)$ belongs to $C^{0, p}\left(\Gamma^{1}\right)$ in $s$ uniformly with respect to $\sigma \in \Gamma$, where $p=\min \{1 / 2, \lambda\}$. Furthermore, taking into account (10c) we have $\Phi_{1}(s) \in$ $C^{0, p}\left(\Gamma^{1}\right)$. Consequently, from equation (18) we can conclude the following assertion.

Lemma. Let $\Gamma^{1} \in C^{2, \lambda}, \Gamma^{2} \in C^{1, \lambda}, \lambda \in(0,1]$, and $\Phi(s) \in C^{0, p}\left(\Gamma^{1}\right) \cap C^{0}\left(\Gamma^{2}\right)$, where $p=\min \{\lambda, 1 / 2\}$. If $\mu_{*}(s)$ from $C^{0}(\Gamma)$ satisfies equation (18), then $\mu_{*}(s)$ belongs to $C^{0, p}\left(\Gamma^{1}\right) \cap C^{0}\left(\Gamma^{2}\right)$.

The condition $\Phi(s) \in C^{0, p}\left(\Gamma^{1}\right) \cap C^{0}\left(\Gamma^{2}\right)$ holds if conditions (3) hold.

Hence below we will seek $\mu_{*}(s)$ from $C^{0}(\Gamma)$.

Consider equation (18). The integral operator

$$
\int_{\Gamma} \mu_{*}(\sigma) Q^{-1}(\sigma) A_{2}(s, \sigma) d \sigma=\int_{\Gamma^{1}} \mu_{*}(\sigma) Q_{1}^{-1}(\sigma) A_{2}(s, \sigma) d \sigma+\int_{\Gamma^{2}} \mu_{*}(\sigma) A_{2}(s, \sigma) d \sigma
$$

is compact from $C^{0}(\Gamma)$ into $C^{0}\left(\Gamma^{2}\right)$. Indeed, using Arzela's theorem one can verify that the first term is a compact operator from $C^{0}\left(\Gamma^{1}\right)$ into $C^{0}\left(\Gamma^{2}\right)$, because $A_{2}(s, \sigma) \in$ $C^{0}\left(\Gamma^{2} \times \Gamma^{1}\right)$. The second term is a compact operator from $C^{0}\left(\Gamma^{2}\right)$ into $C^{0}\left(\Gamma^{2}\right)$, because $A_{2}(s, \sigma)$ is a polar kernel [17]; i.e., it has a weak singularity as $s=\sigma \in \Gamma^{2}$ and it is continuous if $s \neq \sigma \quad\left(s, \sigma \in \Gamma^{2}\right)$. Furthermore, using Arzela's theorem one can show that the integral operator $\int_{\Gamma} \mu_{*}(\sigma) Q^{-1}(\sigma) A_{1}(s, \sigma) d \sigma$ is compact from $C^{0}(\Gamma)$ into $C^{0}\left(\Gamma^{1}\right)$ since $A_{1}(s, \sigma) \in C^{0}\left(\Gamma^{1} \times \Gamma\right)$. Therefore the integral operator from (18):

$$
\mathbf{A} \mu_{*}(s)=\int_{\Gamma} \mu_{*}(\sigma) Q^{-1}(\sigma) A(s, \sigma) d \sigma
$$

is a compact operator mapping $C^{0}(\Gamma)$ into itself. Therefore, $(18)$ is a Fredholm equation of the second kind and index zero in the Banach space $C^{0}(\Gamma)$.

Let us show that if $\mu_{*}^{0}(s)$ is a solution of the homogeneous equation (18) from $C^{0}(\Gamma)$, then it is the trivial solution, i.e., $\mu_{*}^{0}(s) \equiv 0$. Let $\mu_{*}^{0}(s) \in C^{0}(\Gamma)$ be a solution of the homogeneous equation (18). According to the lemma, $\mu_{*}^{0}(s) \in C^{0, p}\left(\Gamma^{1}\right) \cap C^{0}\left(\Gamma^{2}\right), \quad p=$ $\min \{\lambda, 1 / 2\}$. Therefore the function $\mu^{0}(s)=\mu_{*}^{0}(s) Q^{-1}(s) \in C_{1 / 2}^{p}\left(\Gamma^{1}\right) \cap C^{0}\left(\Gamma^{2}\right)$ converts the homogeneous equations (12), (17) into identities. Using the homogeneous identity (17), we check that $\mu^{0}(s)$ satisfies conditions (5). Furthermore, acting on the homogeneous identity (17) with a singular operator with the kernel $(s-t)^{-1}$, we find that $\mu^{0}(s)$ satisfies the homogeneous equation (13). Consequently, $\mu^{0}(s)$ satisfies the homogeneous equations (11). On the basis of Theorem 2, the function $u\left[0, \mu^{0}\right](x)=w\left[\mu^{0}\right](x)+h_{2}\left[\mu^{0}\right](x)$ given by $(6),(7)$ is a solution of the homogeneous problem $\mathbf{U}$. According to Theorem 1 , 
$\left(w\left[\mu^{0}\right](x)+h_{2}\left[\mu^{0}\right](x)\right) \equiv 0, x \in \mathcal{D} \backslash \Gamma^{1}$. Using the limit formulas for tangential derivatives of an angular potential [1, 5, [12, Appendix], we obtain

$$
\begin{gathered}
\lim _{x \rightarrow x(s) \in\left(\Gamma^{1}\right)^{+}} \frac{\partial}{\partial \tau_{x}}\left(w\left[\mu^{0}\right](x)+h_{2}\left[\mu^{0}\right](x)\right) \\
-\lim _{x \rightarrow x(s) \in\left(\Gamma^{1}\right)^{-}} \frac{\partial}{\partial \tau_{x}}\left(w\left[\mu^{0}\right](x)+h_{2}\left[\mu^{0}\right](x)\right)=\mu^{0}(s) \equiv 0, \quad s \in \Gamma^{1} .
\end{gathered}
$$

Hence, $\left(w\left[\mu^{0}\right](x)+h_{2}\left[\mu^{0}\right](x)\right)=\left(w_{2}\left[\mu^{0}\right](x)+h_{2}\left[\mu^{0}\right](x)\right) \equiv 0, \quad x \in \mathcal{D}$, and $\mu^{0}(s)$ satisfies (11b), which takes the form

$$
\begin{gathered}
\frac{1}{2} \mu^{0}(s)-\frac{1}{2 \pi} \int_{\Gamma^{2}} \mu^{0}(\sigma) \frac{\partial}{\partial \mathbf{n}_{y}} \ln |x(s)-y(\sigma)| d \sigma \\
+h_{2}\left[\mu^{0}\right](x(s))=0, \quad s \in \Gamma^{2}
\end{gathered}
$$

where $h_{2}[\mu](x)$ is specified in (7b). The Fredholm equation (19) arises when solving the homogeneous Dirichlet problem for harmonic functions in the exterior domain $\mathcal{D}$ by the double layer potential with the sum of point sources placed inside the curves $\Gamma_{1}^{2}, \ldots, \Gamma_{N_{2}}^{2}$. The equation (19) has only the trivial solution $\mu^{0}(s) \equiv 0$ in $C^{0}\left(\Gamma^{2}\right)$. This is shown in the appendix.

Consequently, if $s \in \Gamma$, then $\mu^{0}(s) \equiv 0, \quad \mu_{*}^{0}(s)=\mu^{0}(s) Q^{-1}(s) \equiv 0$. Thus, the homogeneous Fredholm equation (18) has only the trivial solution in $C^{0}(\Gamma)$.

We have proved the following assertion.

Theorem 3. If $\Gamma^{1} \in C^{2, \lambda}, \Gamma^{2} \in C^{1, \lambda}, \lambda \in(0,1]$, then (18) is a Fredholm equation of the second kind and index zero in the space $C^{0}(\Gamma)$. Moreover, equation (18) has a unique solution $\mu_{*}(s) \in C^{0}(\Gamma)$ for any $\Phi(s) \in C^{0}(\Gamma)$.

As a consequence of Theorem 3 and the lemma we obtain

Corollary. If $\Gamma^{1} \in C^{2, \lambda}, \Gamma^{2} \in C^{1, \lambda}, \lambda \in(0,1]$, then equation (18) has a unique solution $\mu_{*}(s) \in C^{0, p}\left(\Gamma^{1}\right) \cap C^{0}\left(\Gamma^{2}\right)$, for any $\Phi(s) \in C^{0, p}\left(\Gamma^{1}\right) \cap C^{0}\left(\Gamma^{2}\right)$, where $p=\min \{\lambda, 1 / 2\}$.

We recall that $\Phi(s)$ belongs to the class of smoothness required in the corollary if $f(s) \in C^{0, \lambda}\left(\Gamma^{1}\right) \cap C^{0}\left(\Gamma^{2}\right)$. As mentioned above, if $\mu_{*}(s) \in C^{0, p}\left(\Gamma^{1}\right) \cap C^{0}\left(\Gamma^{2}\right)$ is a solution of $(18)$, then $\mu(s)=\mu_{*}(s) Q^{-1}(s) \in C_{1 / 2}^{p}\left(\Gamma^{1}\right) \cap C^{0}\left(\Gamma^{2}\right)$ is a solution of system (5), (11). We obtain the following statement.

Theorem 4. If $\Gamma^{1} \in C^{2, \lambda}, \Gamma^{2} \in C^{1, \lambda}, \lambda \in(0,1]$, then the system of equations (5), (11) has a solution $\mu(s) \in C_{1 / 2}^{p}\left(\Gamma^{1}\right) \cap C^{0}\left(\Gamma^{2}\right), p=\min \{1 / 2, \lambda\}$, for any $f(s) \in C^{0, \lambda}\left(\Gamma^{1}\right) \cap$ $C^{0}\left(\Gamma^{2}\right)$. Moreover, this solution is expressed by the formula $\mu(s)=\mu_{*}(s) Q^{-1}(s)$, where $\mu_{*}(s) \in C^{0, p}\left(\Gamma^{1}\right) \cap C^{0}\left(\Gamma^{2}\right)$ is the unique solution of the Fredholm equation (18) in $C^{0}(\Gamma)$.

REMark. The solution of the system (5), (11) ensured by Theorem 4 is unique in the space $C_{1 / 2}^{p_{o}}\left(\Gamma^{1}\right) \cap C^{0}\left(\Gamma^{2}\right)$ for any $p_{o} \in(0, p]$. More precisely, the system (5), (11) has at most one solution in the space $C_{q}^{\omega}\left(\Gamma^{1}\right) \cap C^{0}\left(\Gamma^{2}\right)$ for any $\omega \in(0,1]$ and $q \in[0,1)$. The proof of this fact almost coincides with the proof of Theorem 3 . 
According to (10), $f(s)$ belongs to $C^{0, \lambda}\left(\Gamma^{1}\right) \cap C^{0}\left(\Gamma^{2}\right)$ if (3) holds. Therefore, the condition $f(s) \in C^{0, \lambda}\left(\Gamma^{1}\right) \cap C^{0}\left(\Gamma^{2}\right)$ in Theorem 4 can be replaced by the condition that (3) holds. On the basis of Theorem 2 and Theorem 4 we arrive at the final result.

Theorem 5. If $\Gamma^{1} \in C^{2, \lambda}, \Gamma^{2} \in C^{1, \lambda}$ and condition (3) holds, then the solution of the problem $\mathbf{U}$ exists, belongs to the class $\mathbf{K}$ and is given by (6), where $\nu(s)$ is defined in (9) and $\mu(s)$ is a solution of system (5), (11) from $C_{1 / 2}^{p}\left(\Gamma^{1}\right) \cap C^{0}\left(\Gamma^{2}\right), p=\min \{1 / 2, \lambda\}$, ensured by Theorem 4 .

It can be checked directly that the solution of the problem $\mathbf{U}$ constructed in Theorem 5 satisfies condition (1) with $\epsilon=-1 / 2$. Explicit expressions for the singularities of the solution gradient at the endpoints of the open curves will be presented in the next section.

Theorem 5 ensures the existence of a classical solution of the problem $\mathbf{U}$ when $\Gamma^{1} \in$ $C^{2, \lambda}, \Gamma^{2} \in C^{1, \lambda}$, and condition (3) holds. The uniqueness of the classical solution follows from Theorem 1. On the basis of our consideration we suggest the following scheme for solving the problem $\mathbf{U}$. At first, we find the unique solution $\mu_{*}(s)$ of the Fredholm equation (18) from $C^{0}(\Gamma)$. This solution automatically belongs to $C^{0, p}\left(\Gamma^{1}\right) \cap$ $C^{0}\left(\Gamma^{2}\right), p=\min \{\lambda, 1 / 2\}$. Secondly, we construct the solution of equations (5), (11) from $C_{1 / 2}^{p}\left(\Gamma^{1}\right) \cap C^{0}\left(\Gamma^{2}\right)$ by the formula $\mu(s)=\mu_{*}(s) Q^{-1}(s)$. Finally, substituting $\nu(s)$ from (9) and $\mu(s)$ in (6), we obtain the solution of the problem $\mathbf{U}$.

5. The behaviour of the gradient of the solution at the tips of the cuts $\Gamma^{1}$. In the present section by $u(x)=u[\nu, \mu](x)$ we denote the solution of problem $\mathbf{U}$ constructed in the previous section. The integral representation for $u(x)$ obtained in Theorem 5 enables us to derive explicit formulas for the singularities of $\nabla u$ at the tips of the cuts $\Gamma^{1}$. It follows from the definition of the class $\mathbf{K}$ that the gradient of the solution of the problem $\mathbf{U}$ might be unbounded at the endpoints of $\Gamma^{1}$, where the estimate (1) holds with $\epsilon=-1 / 2$. Our aim now is to investigate in detail the behaviour of $\nabla u(x)$ at the endpoints of $\Gamma^{1}$. Let $x(d)$ be one of these points $\left(d=a_{n}^{1}\right.$ or $d=b_{n}^{1}$, where $\left.n=1, \ldots, N_{1}\right)$. In the neighbourhoods of $x(d)$ we introduce the polar system of coordinates

$$
x_{1}=x_{1}(d)+|x-x(d)| \cos \varphi, \quad x_{2}=x_{2}(d)+|x-x(d)| \sin \varphi .
$$

We will assume that $\varphi \in(\alpha(d), \alpha(d)+2 \pi)$ if $d=a_{n}^{1}$, and $\varphi \in(\alpha(d)-\pi, \alpha(d)+\pi)$ if $d=b_{n}^{1}$. Recall that $\alpha(s)$ is the angle between the $O x_{1}$ axis and the tangent vector $\tau_{x}$ drawn at the point $x(s) \in \Gamma$. Hence, $\alpha(d)=\alpha\left(a_{n}^{1}+0\right)$ if $d=a_{n}^{1}$, and $\alpha(d)=\alpha\left(b_{n}^{1}-0\right)$ if $d=b_{n}^{1}$. Thus, the angle $\varphi$ varies continuously in a neighbourhood of $x(d)$ cut along the contour $\Gamma^{1}$.

Let $\mu_{1}(s)=\mu(s)|s-d|^{1 / 2}=Q^{-1}(s) \mu_{*}(s)|s-d|^{1 / 2}$ and put $\mu_{1}(d)=\mu_{1}\left(a_{n}^{1}\right)=\mu_{1}\left(a_{n}^{1}+0\right)$ if $d=a_{n}^{1}, \mu_{1}(d)=\mu_{1}\left(b_{n}^{1}\right)=\mu_{1}\left(b_{n}^{1}-0\right)$ if $d=b_{n}^{1}$.

Recall that $X$ is the set of endpoints of $\Gamma^{1}$. The following theorem is easily proved using the results obtained in [5] and using the properties of Cauchy type integrals near the endpoints of the integration line given in [16, section 22], [2, section 8]. 
Theorem 6 . Let $x \rightarrow x(d) \in X$. Then in the neighbourhood of the point $x(d)$, the derivatives of the solution of the problem $\mathbf{U}$ satisfy the relations

$$
\begin{gathered}
\frac{\partial}{\partial x_{1}} u(x)=-(-1)^{m} \frac{\mu_{1}(d)}{2|x-x(d)|^{1 / 2}} \sin \gamma \\
-(-1)^{m} \frac{\nu(d)}{2 \pi}[\ln |x-x(d)| \cos \alpha(d)+\varphi \sin \alpha(d)]+O(1), \\
\frac{\partial}{\partial x_{2}} u(x)=(-1)^{m} \frac{\mu_{1}(d)}{2|x-x(d)|^{1 / 2}} \cos \gamma \\
+(-1)^{m} \frac{\nu(d)}{2 \pi}[-\ln |x-x(d)| \sin \alpha(d)+\varphi \cos \alpha(d)]+O(1),
\end{gathered}
$$

where $m=0, \gamma=[\varphi+\alpha(d)-\pi] / 2$ if $d=a_{n}^{1}$, and $m=1, \gamma=[\varphi+\alpha(d)] / 2$ if $d=b_{n}^{1}$, and where $O(1)$ denotes functions which are continuous at the point $x(d)$. Furthermore, the functions denoted by $O(1)$ are continuous in the neighbourhood of the point $x(d)$, cut along the contour $\Gamma^{1}$.

This theorem establishes the following curious fact. In the general case, the derivatives of the solution of the problem $\mathbf{U}$ near the endpoint $x(d)$ of the contour $\Gamma^{1}$ behave as $O\left(|x-x(d)|^{-1 / 2}\right)+O\left(\ln |x-x(d)|^{-1}\right)$. However, if $\mu_{1}(d)=\nu(d)=0$, then $\nabla u(x)$ will be bounded and even continuous at the endpoint $x(d)$ of $\Gamma^{1}$. This effect of disappearence of singularities happens for certain functions $F^{ \pm}(s), F(s)$ given in the boundary condition (2a), since the condition $\mu_{1}(d)=\nu(d)=0$ specifies restrictions on these functions.

Appendix. Here we prove the following assertion.

Proposition A. If $\Gamma^{2} \in C^{1, \lambda}, \lambda \in(0,1]$, then there exists only the trivial solution of the homogeneous Fredholm equation (19) in $C^{0}\left(\Gamma^{2}\right)$.

Proof. Let $\mu^{0}(s) \in C^{0}\left(\Gamma^{2}\right)$ be a nontrivial solution of the homogeneous equation (19). The kernel of the integral term in (19) has a weak singularity. It can be shown with the help of [16, Sec. 51] that the integral term in (19) belongs to $C^{0, \lambda / 4}\left(\Gamma^{2}\right)$ in $s$; therefore $\mu^{0}(s) \in C^{0, \lambda / 4}\left(\Gamma^{2}\right)$. Now we consider the function

$$
g\left[\mu^{0}\right](x)=w_{2}\left[\mu^{0}\right](x)+h_{2}\left[\mu^{0}\right](x),
$$

where $w_{2}\left[\mu^{0}\right](x)$ and $h_{2}\left[\mu^{0}\right](x)$ were introduced in (7a), (7b). The function $g\left[\mu^{0}\right](x)$ belongs to $C^{0}(\overline{\mathcal{D}}) \cap C^{2}(\mathcal{D})$ and satisfies the following homogeneous Dirichlet problem for the Laplace equation:

$$
\Delta g=0 \text { in } \mathcal{D},\left.\quad g\right|_{\Gamma^{2}}=0, \quad|g| \leq \text { const in } \overline{\mathcal{D}} .
$$

Indeed, substituting $g\left[\mu^{0}\right](x)$ in the boundary condition, we get the identity (19). According to the uniqueness theorem for the Dirichlet problem, we have

$$
g\left[\mu^{0}\right](x) \equiv 0, \quad x \in \overline{\mathcal{D}} .
$$

Therefore, letting $|x| \rightarrow \infty$ in the expression for $g\left[\mu^{0}\right](x)$, we obtain

$$
\int_{\Gamma^{2}} \mu^{0}(\sigma) d \sigma=0 .
$$


We consider the function

$$
\begin{gathered}
g^{*}\left[\mu^{0}\right](x)=-\frac{1}{2 \pi}\left[-\int_{\Gamma^{2}} \mu^{0}(\sigma) \frac{\partial}{\partial \sigma} \ln |x-y(\sigma)| d \sigma\right. \\
\left.+\sum_{k=2}^{N_{2}} \int_{\Gamma_{k}^{2}} \mu^{0}(\sigma) d \sigma V\left(x, Y_{k}\right)-\left(\int_{\Gamma^{2}} \mu^{0}(\sigma) d \sigma-\int_{\Gamma_{1}^{2}} \mu^{0}(\sigma) d \sigma\right) V\left(x, Y_{1}\right)\right],
\end{gathered}
$$

where $V(x, y)$ is the kernel of the angular potential from (4). The function $g^{*}\left[\mu^{0}\right](x)$ is the harmonic conjugate to $g\left[\mu^{0}\right](x)$, i.e., the Cauchy-Riemann relations $\partial_{x_{1}} g=\partial_{x_{2}} g^{*}, \partial_{x_{2}} g=$ $-\partial_{x_{1}} g^{*}$ hold. Consequently, $g^{*}\left[\mu^{0}\right](x) \equiv$ Const in $\mathcal{D}$. It is clear from (A4), that $g^{*}\left[\mu^{0}\right](x)$ is a many-valued function, because $V\left(x, Y_{k}\right)$ are many-valued functions $\left(k=1, \ldots, N_{2}\right)$. Indeed, when passing around the point $Y_{k}$ the value of the function $V\left(x, Y_{k}\right)$ changes for $2 \pi$. Evidently, $g^{*}\left[\mu^{0}\right](x)$ can be constant in $\mathcal{D}$ only if $g^{*}\left[\mu^{0}\right](x)$ is single-valued. In order for $g^{*}\left[\mu^{0}\right](x)$ to be single-valued, the following $N_{2}$ conditions must hold:

$$
\begin{gathered}
\int_{\Gamma_{k}^{2}} \mu^{0}(\sigma) d \sigma=0, \quad k=2, \ldots, N_{2}, \\
\int_{\Gamma^{2}} \mu^{0}(\sigma) d \sigma-\int_{\Gamma_{1}^{2}} \mu^{0}(\sigma) d \sigma=0 .
\end{gathered}
$$

Along with (A3) we obtain

$$
\int_{\Gamma_{k}^{2}} \mu^{0}(\sigma) d \sigma=0, \quad k=1, \ldots, N_{2} .
$$

Under these conditions, $g^{*}\left[\mu^{0}\right](x)$ takes the form of the modified single-layer potential [16]

$$
g^{*}\left[\mu^{0}\right](x) \equiv w_{2}^{*}\left[\mu^{0}\right](x)=\frac{1}{2 \pi} \int_{\Gamma^{2}} \mu^{0}(\sigma) \frac{\partial}{\partial \sigma} \ln |x-y(\sigma)| d \sigma,
$$

and $g\left[\mu^{0}\right](x)$ transforms to the ordinary double-layer potential

$$
\begin{gathered}
g\left[\mu^{0}\right](x) \equiv w_{2}\left[\mu^{0}\right](x)=-\frac{1}{2 \pi} \int_{\Gamma^{2}} \mu^{0}(\sigma) \frac{\partial}{\partial \mathbf{n}_{y}} \ln |x-y(\sigma)| d \sigma \\
\in C^{0}\left(\overline{R^{2} \backslash \Gamma^{2}}\right) \cap C^{2}\left(R^{2} \backslash \Gamma^{2}\right) .
\end{gathered}
$$

The potentials (A6) and (A7) are connected by the Cauchy-Riemann relations in $R^{2} \backslash \Gamma^{2}$. Because of $\mu^{0}(s) \in C^{0, \lambda / 4}\left(\Gamma^{2}\right)$, the potential (A6) is a harmonic function, which belongs to $C^{0}\left(R^{2}\right) \cap C^{2}\left(R^{2} \backslash \Gamma^{2}\right)$ (see [16] for details). Note that (A6) is continuous when passing through $\Gamma^{2}$ and is represented on $\Gamma^{2}$ by a singular integral (for this we have stressed that $\mu^{0}(s)$ is a Hölder continuous function).

As stated above, $w_{2}^{*}\left[\mu^{0}\right](x)$ in $\overline{\mathcal{D}}$ is equal to a constant, which is equal to zero due to the behaviour of this potential at infinity, so that $w_{2}^{*}\left[\mu^{0}\right](x) \equiv 0$ in $\overline{\mathcal{D}}$. 
We consider the internal domain $\mathcal{D}_{k}$ bounded by $\Gamma_{k}^{2} \quad\left(k=1, \ldots, N_{2}\right)$. In this domain the potential (A6) satisfies the Dirichlet problem

$$
\Delta w_{2}^{*}=0 \text { in } \mathcal{D}_{k},\left.\quad w_{2}^{*}\right|_{\Gamma_{k}^{2}}=0,
$$

which has the unique solution

$$
w_{2}^{*}\left[\mu^{0}\right](x) \equiv 0, \quad x \in \overline{\mathcal{D}_{k}}\left(k=1, \ldots, N_{2}\right) .
$$

It follows from the Cauchy-Riemann relations and the smoothness of the double-layer potential that

$$
w_{2}\left[\mu^{0}\right](x) \equiv c_{k}, \quad x \in \overline{\mathcal{D}_{k}}, \quad k=1, \ldots, N_{2},
$$

where $c_{1}, \ldots, c_{N_{2}}$ are constants. Using (A2) and the jump relation for the double-layer potential $w_{2}\left[\mu^{0}\right](x)$ on $\Gamma^{2}$, we get

$$
\left.\mu^{0}(s)\right|_{\Gamma_{k}^{2}} \equiv-c_{k}, \quad k=1, \ldots, N_{2} .
$$

According to (A5), $c_{k}=0, k=1, \ldots, N_{2}$, and therefore

$$
\left.\mu^{0}(s)\right|_{\Gamma_{k}^{2}} \equiv 0, \quad k=1, \ldots, N_{2} .
$$

Consequently,

$$
\mu^{0}(s) \equiv 0 \text { on } \Gamma^{2} \text {. }
$$

Hence, the homogeneous equation (19) has only the trivial solution. The proof is completed.

Because (19) is a Fredholm equation of the second kind and index zero, the following corollary holds.

Corollary A. If $\Gamma^{2} \in C^{1, \lambda}, \lambda \in(0,1]$, then the inhomogeneous Fredholm equation (19) is uniquely solvable in $C^{0}\left(\Gamma^{2}\right)$ for any right-hand side from $C^{0}\left(\Gamma^{2}\right)$.

The inhomogeneous equation (19) is a particular case of (11b) if the exterior domain $\mathcal{D}$ does not contain cuts.

Acknowledgment. This research was supported by the RFBR grants No. 05-0100050, 07-01-00029.

\section{REFERENCES}

[1] Gabov S.A. An angular potential and its applications. Math. U.S.S.R. Sbornik, 1977, v.32, No.4, pp. 423-436.

[2] Gakhov F.D. Boundary value problems. Pergamon Press, Oxford; Addison-Wesley, Reading, Mass., 1966. MR0198152 (33:6311)

[3] Gilbarg D., Trudinger N.S. Elliptic partial differential equations of second order. Springer, Berlin, 1983. MR0737190 (86c:35035)

[4] Krutitskii P.A. On the electric current from electrodes in a magnetized semiconductor film. IMA J. Appl. Math., 1998, v.60, pp. 285-297. MR1653863 (99h:78026)

[5] Krutitskii P.A. The Dirichlet problem for the Helmholtz equation outside cuts in a plane. Comp. Math. Math. Phys., 1994, v.34, No.8/9, pp. 1073-1090. MR1300397(95f:35046)

[6] Krutitskii P.A. The Neumann problem for the Helmholtz equation outside cuts in the plane. Comput. Math. Math. Phys., 1994, No.11, pp. 1421-1431.

[7] Krutitskii P.A. The 2-Dimensional Dirichlet Problem in an External Domain with Cuts. Zeitscr. Analysis u. Anwend., 1998, v.17, No.2, pp. 361-378. MR1632551 (99f:35036) 
[8] Krutitskii P.A. The Neumann problem in a 2-D exterior domain with cuts and singularities at the tips. J. Differential Equations, 2001, v.176, No.1, pp. 269-289. MR.1861190 (2002g:35056)

[9] Krutitskii P.A. Wave propagation in a 2-D external domain bounded by closed and open curves. Nonlinear Analysis, Theory, Methods and Applications, 1998, v.32, No.1, pp. 135-144. MR.1491619 (98m:35040)

[10] Krutitskii P.A. Wave scattering in a 2-D exterior domain with cuts: The Neumann problem. ZAMM, 2000, v.80, No.8, pp. 535-546. MR.1775291 (2001d:35037)

[11] Krutitskii P.A. An explicit solution of the pseudo-hyperbolic initial boundary value problem in a multiply connected region. Mathematical Methods in the Applied Sciences, 1995, v.18, No.11, pp. 897-925. MR 1346665 (97d:76010a)

[12] Krutitskii P.A. The oblique derivative problem for the Helmholtz equation and scattering tidal waves. Proceedings of the Royal Society of London, ser. A, 2001, v.457, pp. 1735-1755. MR1850934 (2002g:35051)

[13] Medkova D. Solution of the Dirichlet problem for the Laplace equation. Applications of Mathematics, 1999, v.44, pp. 143-168. MR1667634(2000a:35040)

[14] Lifanov I.K. Singular integral equations and discrete vortices. VSP, Utrecht, 1996. MR1451377 (98g:65130)

[15] Gunter N.M. Potential theory and its application to the basic problems of mathematical physics. GITTL, Moscow, 1953.

[16] Muskhelishvili N.I. Singular integral equations, Nauka, Moscow, 1968 (3rd Russian edition). MR0355495 (50:7969)

[17] Vladimirov V.S. Equations of Mathematical Physics, Marcel Dekker, N.Y., 1971. MR0352661 $(50: 5148)$

[18] Durand M. Layer potentials and boundary value problems for the Helmholtz equation in the complement of a thin obstacle. Math. Meth. Appl. Sci., 1983, v.5, pp. 389-421. MR0716663 (84k:35040)

[19] Panasyuk, V.V., Savruk, M.P., Nazarchuk, Z.T. The method of singular integral equations in two-dimensional diffraction problems, Naukova Dumka, Kiev, 1984. (in Russian). MR0780185 (86i:45009)

[20] Tuchkin Y. A., Scattering of waves by an unclosed cylindrical screen of arbitrary profile with Neumann boundary condition. Dokl. Akad. Nauk SSSR, 293 (1987), pp. 343-345. (in Russian). MR0884044 (89d:78033) 